\title{
The Food-Borne Micro RNA and its Controversy on Human Health
}

\author{
Keshab Bhattarai* and Sudhashree Adhikari \\ Nutritional Science Laboratory, The United Graduate School of Agricultural Sciences, Ehime University, Japan \\ *Corresponding author: Keshab Bhattarai, Nutritional Science Laboratory, Graduate School of Agriculture, Ehime University, 3-5-7 Tarumi, \\ Matsuyama, 790-8566, Japan
}

Submission: April 19, 2018; Published: May 31, 2018

\section{Editorial}

A micro-RNA (mi-RNA) is a small non-coding RNA molecule containing about 21-25 nucleotides. Micro-RNAs are partially complementary to one or more messenger RNA (mRNA) molecules, and their main function is to down regulate the gene expression in a variety of manners, including translational repression, mRNA cleavage, and deadenylation. This mechanism relies on the seed region of nucleotide sequence of mi-RNAs that bind to target mRNA [1]. Imperfect pairing of sequences in the seed region in mi-RNA to mRNA impairs gene down- regulation at the protein or RNA level [2]. The mRNA degradation occurs if the mi-RNA nucleotide sequence has a high degree of complementarities to the mRNA sequence $[3,4]$. Most of the circulating mi-RNAs exist in packaged exosomes $[5,6]$. Exosomes are the extracellular vesicles containing variety of compounds, lipids, proteins including mRNAs, micro-RNAs (miRNAs), and other non-coding RNAs (ncRNAs) [7,8]. Exosomes are not only play essential roles in cell-to-cell communication but also in the role of protection against enzymatic and non-enzymatic degradation of cargos.

In the past, endogenous-mi-RNA has been considered as a regulator of the expression of genes within the host. Evidence suggests that the endogenous synthesis of mi-RNAs can be altered by the dietary bioactive components. Polyphenolic compounds from food sources like fruits and beverages such as tea, coffee, and wine can modulate the expression of mi-RNAs that regulate mRNA which are involved in various biological functions, such as apoptosis, inflammation, lipid metabolism, and cell migration [9].

Interestingly, mature exogenous mi-RNAs may also be obtained from dietary sources [10]. Recently it has been claimed that the miRNA from the food-borne especially plants and animals-origin miRNAs are bio available and can affect gene expression. For instance, Zhang et al. [11] reported that orally administered exogenous plant mi-RNAs through food intake are present in the sera and tissues of various animals and finally can regulate the expression of target genes in mammals. They reported that osa-MIR-168a (found in rice) decreased low- density lipoprotein receptor adapter protein 1 mRNA in the mouse liver. The plant-origin mi-RNAs were also identified by the Lukasik \& Zielenkiewicz [12] reported that some plant mi-RNA molecules are abundant in human and porcine breast milk exosomes. However, studies regarding on the bioavailability of plant-origin mi-RNAs are also controversial. Snow et al. [13] concluded that "Horizontal delivery of micro-RNAs via typical dietary ingestion is neither a robust nor a frequent mechanism to maintain steady-state micro-RNA levels in a variety of model animal organisms, thus defining the biological limits of these molecules in vivo". Dickinson et al. [14] did not detect the oral bioavailability of plant micro-RNAs after feeding in mice.

Animal-origin mi-RNAs are bio available and can affect gene expression in mice and human [15]. The main discovery of the Zempleni group on dietary mi-RNA concluded that

A. humans absorb biologically meaningful amounts of miRNAs from nutritionally relevant doses of cow's milk,

B. Milk mi-RNAs are delivered to peripheral human tissues,

C. physiological concentrations of milk mi-RNAs affect human gene expression in vivo and in cell cultures, and

D. Endogenous synthesis of mi-RNAs does not compensate for dietary mi-RNAs deficiency in mice" [16].

In their plant-origin mi-RNAs study, they did not detect the Brassica-specific mi-RNAs in a broccoli sprout-feeding study.

There are some evidences that mi-RNAs (endogenous as well as exogenous) are contained in exosomes, providing protection against degradation from acidic environment of intestine and adequately stable to pass through the gastrointestinal (GI) tract and enter into circulation without losing its functionality. This intestinal uptake of micro-RNAs contained exosomes is mediated by endocytosis where the protein/protein recognition plays an important role in the intestinal uptake in humans and rats [17]. The glycosylated proteins are important to the endocytosis of food-borne exosomes. The more compatibility of glycoprotein's with the receptors on the apical surface of mammalian cells the more bioavailability of miRNAs. When compared to animal-origin mi-RNAs, bioavailability 
of plant-origin mi-RNAs is quite low, that could be due to the poor compatibility of plant vesicles [17].

To explore more on the exogenous mi-RNAs and its effect on peripheral gene expression on human, one should understand the information of mi-RNA molecules through the computational analytical approaches; more experiments in characterization of intestinal mi-RNA transport mechanisms and alter the gene expression through binding to mRNA in hosts; and molecular interaction of the glycoprotein's involved in the endocytosis of dietary micro-RNAs.

\section{References}

1. Lewis BP, Burge CB, Bartel DP (2005) Conserved seed pairing, often flanked by adenosines, indicates that thousands of human genes are microRNA targets. Cell 120(1): 15-20.

2. Wang $X$ (2014) Composition of seed sequence is a major determinant of microRNA targeting patterns. Bioinformatics 30(10): 1377-1383.

3. Ameres SL, Zamore PD (2013) Diversifying microRNA sequence and function. Nat Rev Mol Cell Biol 14(8): 475-488.

4. Rana TM (2007) Illuminating the silence: understanding the structure and function of small RNAs. Nat Rev Mol Cell Biol 8(1): 23-36.

5. Simpson RJ, Lim JW, Mortiz RL, Mathivanan S (2009) Exosomes: Proteomic insights and diagnostic potential. Expert Rev Proteomics 6(3): 267-283.

6. Kosaka N, Iguchi H, Ochiya T (2010) Circulating microRNA in body fluid: A new potential biomarker for cancer diagnosis and prognosis. Cancer Sci 101(10): 2087-2092.

7. Sato Kuwabara Y, Melo SA, Soares FA, Calin GA (2015) The fusion of two worlds: non-coding RNAs and extracellular vesicles - diagnostic and therapeutic implications (Review). Int J Oncol 46(1): 17-27.

8. Zempleni J, Aguilar Lozano A, Sadri M, Sukreet S, Manca S, et al. (2017)
Biological activities of extracellular vesicles and their cargos from bovine and human milk in humans and implications for infants. J Nutr 147(1): 3-10.

9. Milenkovic D, Jude B, Morand C (2013) miRNA as molecular target of poly-phenols underlying their biological effects. Free Radic Biol Med 64: 40-51.

10. Baier SR, Nguyen C, Xie F, Wood JR, Zempleni J (2014) MicroRNAs are absorbed in biologically meaningful amounts from nutritionally relevant doses of cow's milk and affect gene expression in peripheral blood mononuclear cells, HEK-293 kidney cell cultures, and mouse livers. J Nutr 144(10): 1495-1500.

11. Zhang L, Hou D, Chen X, Li D, Zhu LX, et al. (2012) Exogenous plant MIR168a specifically targets mammalian LDLRAP1: evidence of crosskingdom regulation by microRNA. Cell Res 22: 107-126.

12. Lukasik A, Zielenkiewicz P (2014) In silico identification of plant miRNAs in mammalian breast milk exosomes-A small step for- ward? PLoS ONE 9: e99963.

13. Snow JW, Hale AE, Isaacs SK, Baggish AL, Chan SY (2013) Ineffective delivery of diet-derived microRNAs to recipient animal organisms. RNA Biol 10(7): 1107-1116

14. Dickinson B, Zhang Y, Petrick JS, Heck G, Ivashuta S, et al. (2013) Lack of detectable oral bioavailability of plant microRNAs after feeding in mice. Nat Biotechnol 31(11): 965-967.

15.Zempleni J, Baier SR, Howard KM, Cui J (2015) Gene regulation by dietary microRNAs. Can J Physiol Pharmacol 93(12): 1097-1102.

16. Baier SR, Nguyen C, Xie F, Wood JR, Zempleni J (2014) MicroRNAs are absorbed in biologically meaningful amounts from nutritionally relevant doses of cow's milk and affect gene expression in peripheral blood mononuclear cells, HEK-293 kidney cell cultures, and mouse livers. J Nutr 144(10): 1495-1500.

17. Wolf T, Baier SR, Zempleni J (2015) The intestinal transport of bovine milk exosomes is mediated by endocytosis in human colon carcinoma caco-2 cells and rat small intestinal IEC-6 cells. J Nutr 145(10): 22012206.

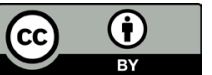

Creative Commons Attribution 4.0 International License

For possible submissions Click Here

\section{Submit Article}

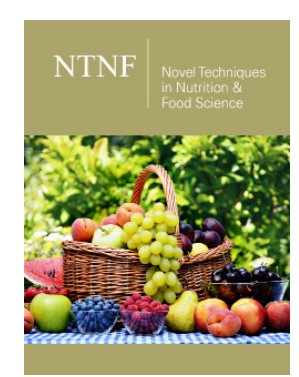

Novel Techniques in Nutrition and Food Science

\section{Benefits of Publishing with us}

- High-level peer review and editorial services

- Freely accessible online immediately upon publication

- Authors retain the copyright to their work

- Licensing it under a Creative Commons license

- Visibility through different online platforms 